\title{
Bipolar Disorder: Definition, Differential Diagnosis, Clinical contexts and Therapeutic approaches
}

\section{Dr. G. Perrotta}

Department of Criminal and Investigative Psychology UNIFEDER

Corresponding Author: Dr. G. Perrotta, Department of Criminal and Investigative Psychology UNIFEDER.

E-mail: giuliosr1984@hotmail.it

Received date: October 19, 2019; Accepted date: November 13, 2019; Published date: November 18, 2019

Citation: Perrotta G. (2019) Bipolar Disorder: Definition, Differential Diagnosis, Clinical contexts and Therapeutic approaches. J. Neuroscience and Neurological Surgery. 5(1); DOI:10.31579/2578-8868/097

Copyright : (C) 2019 Dr. G. Perrotta. This is an open-access article distributed under the terms of The Creative Commons Attribution License, which permits unrestricted use, distribution, and reproduction in any medium, provided the original author and source are credited.

\section{Abstract}

Starting from the general concept of "bipolarity", the present work focuses on the essential aspects of the disorder defining the clinical and diagnostic contexts, laying the foundations for correct differential diagnosis, without neglecting the neural characteristics elaborated in the scientific community. The discussion concludes with the best therapeutic approaches suggested on the subject, hypothesizing that borderline disorder may be the evolution of bipolar disorder.

Key words: bipolar disorder; differential diagnosis; therapeutic approaches; developmental disorders

\section{Contents of the manuscript:}

\section{Definition, differential diagnosis and clinical context of bipolar disorder}

\subsection{Definition, introduction and epidemiological profile}

Bipolar disorder, also known as manic-depressive illness or manicdepressive psychosis, is a disorder characterized by sudden swings in mood and a person's ability to function, without a seemingly justifying cause. Bipolar disorder is therefore characterized by the development of periodic alterations of the level of psychic activation, episodes sometimes interspersed with asymptomatic periods (euthymia, normothermia or normoforìa), sometimes framed at specific intervals of time (as happens in "Seasonal affective disorder"), among other things capable of becoming chronic in protracted phases with deterioration of the individual's condition, which is facing an inexorable decline. In reality, the effective symptomatology is not the most evident manifestation, instead the behavioral consequences are, moreover more easily objective and - from an operational point of view - more useful for diagnostic purposes: bipolar disorder is characterized, in fact, from the alternation of a depressive state and a manic (or hypomanic) state; when there is a simultaneous presence of depressive symptoms and manic symptoms (that is, one is neither wholly depressed nor completely in mania), with the predominance of irritability, anxiety and restlessness, one speaks instead of a mixed state. [1]

From bipolar disorder, however, it must be immediately distinguished from the psychological phenomenon linked to "mood swings from a known cause": these events, such as the loss of a job, the reception of unfortunate news or a stress event, are in themselves sufficient to justify humoral alteration. [2] Not surprisingly, the epidemiological profile speaks of a worldwide percentage around 2, although this datum is probably quite underestimated. [3] Late adolescence and youth are peak years for the onset of bipolar disorder, with a significant onset around the age of 18 (for the cyclothymic disorder 15-20 years); [4] A study also found that in $10 \%$ of cases of bipolar disorder, the beginning of the condition occurred after the age of 50. [5]

\subsection{Etiological profile}

The hypothesized causes for bipolar disorder are heterogeneous and Include biological, genetic and environmental factors.

First, neurochemistry plays a central role: the serotonin deficiency (and in Auctores Publishing -Volume 5(1)-097 www.auctoresonline.org Page - 1 some cases even norepinephrine) seems to be implicated in the onset of depressive symptoms [6], as well as the excess of cortisol. [7]

From a genetic point of view, however, chromosomes 5, 11 and $\mathrm{X}$ are strongly implicated. [8] A genetic variant of the Odz4 gene is undoubtedly associated with this disorder [9], but several genetic linkage studies for the manic syndrome today have led to the discovery of other genes involved: P2RX7, TPH1, HTR2C, TPH, 5HTT and 5HT2A. Recent meta-analyses of linkage studies have revealed the lack of significant variations at a full genomic level, or, using a different methodology, only two significant peaks at the genomic level, on chromosome $6 \mathrm{q}$ and $8 \mathrm{q} 21$. Genetic association studies did not lead to identifying a consistent focus, since each study identified a new locus, without any of the previously identified loci being replicated. [10] [11] Research has revealed a single nucleotide polymorphism in DGKH; [11] a locus in a region rich in genes with extensive Linkage Disequilibrium on chromosome 16p12; [12] and a single nucleotide polymorphism in MYO5B. [13]. A comparison of these studies, combined with a new study, suggested the association with ANK3e CACNA1C, which is currently believed to be related to calcium and sodium channels [14]. Several findings strongly indicate a heterogeneity, with several genes implicated in different families [15]. Several specific studies have found various specific links [16-20]. The advanced age of the parents at the time of conception has been linked in some way to the increased risk of bipolar disorder in the offspring, which is consistent with the hypothesis of the increase of new mutations [21]. A review aimed at identifying the most consistent findings suggested several genes related to serotonin (SLC6A4 and TPH2), dopamine (DRD4 and SLC6A3), glutamate (DAOA and DTNBP1), metabolic pathways involved in cell growth or maintenance (NRG1, DISC1 and BDNF), although it found the possibility of a high risk of false positives in the published literature. It has also been suggested that probably single genes have only a small effect and are involved in some aspect related to bipolar disorder (and a broad aspect of what we call "normal" human behaviour) rather than the disorder itself [22].

From an environmental point of view, finally, the disorder can be triggered in subjects predisposed by influential social events, such as to induce them to create parallel universes in the affective or working spheres, always generating the anxiety of the absence of the other. According to the most reliable hypotheses, the various factors interact causing an imbalance of the functions of the neurotransmitters within some specific areas of the brain, with an alteration in favour of excitatory transmission (episodes of a manic or hypomanic nature) or inhibitory (episodes of character depressive). Psychosocial factors, such as abuse during childhood, deaths and severe 
emotional losses, sentimental shocks and financial or work problems can trigger predisposed subjects the process that gives rise to the disease. The predisposition is, however, correlated with the heredity or to the persistence of strong exogenous stimuli. [1]

\subsection{Clinical context of bipolar disorder [1]}

Although, in clinical psychology, it is recognized that bipolar disorder has three substantial forms, in clinical practice there is no agreement on the number of possible variants: this is because bipolar disorder is conceptualized as a spectrum of disorders that occur in a continuum and the discrimination between the various clinical subtypes of the bipolar spectrum takes place substantially on the basis of the course and the symptomatological connotation of the intercurrent affective phases, also taking into account the possible implications determined by the comorbidities.

As for the DSM-IV-TR, the DSM-V lists three specific subtypes for the disorder in question:

"Type I bipolar disorder". It is diagnosed in the presence of a mixed episode (understood as a morbid affective state generated by the perfect mixture of excitatory symptoms, e.g. delusional thought disorders associated with depressive symptoms or expanded mood and absence of delusional thoughts) or at least one episode of depression major separated by at least one spontaneous manic episode. In most cases, however, manic or mixed episodes are alternated with one or more depressive episodes.

"Type II bipolar disorder". It is diagnosed in the presence of at least one major depressive episode separated by at least one spontaneous hypomanic episode.

"Cyclothymic Disorder". It is diagnosed in the presence of various hypomanic episodes, alternating with periods characterized by the presence of depressive symptoms, which however are not sufficient to diagnose major depression.

"Bipolar disorder not otherwise specified". It is diagnosed when the symptoms presented do not fully meet the diagnostic criteria of the other syndromes. An example is when there is an apparent alternation (very rapid, within a few days) of manic and depressive symptoms that do not meet the minimum duration criteria to define a manic episode or a major depressive episode.

he "mania" is an endogenous psycho-affective excitement syndrome, that is, with an autochthonous genesis, characterized by the morbid, pervasive, protracted and otherwise unjustified exaltation of vital feelings and emotions, as well as instinctive impulses; such a state of pathological relationship is typically associated with a sense of hypertrophic self-esteem, optimism and ease in pursuing one's goals, which underpin feelings of security and power, often flaunted.

In the "manic episode" it is possible to identify three phases: one of onset (euphoria and hyperactivity), one of state (emotional lability, psychomotor agitation and delusions) and one of organization or resolution (psychic disorganization and behavioural bizarre). Among the risk factors for the development of manic excitement in vulnerable individuals is the reduction of sleep time, the use of drugs (antidepressants and glucocorticoids), the abuse of psychostimulant substances (coffee, amphetamines and cocaine).

Usually, the onset phase of the manic episode is more sudden than that of depression: sometimes the mania is announced by prodromal symptoms, eg. early awakenings associated with a sense of well-being, increased energy and renewed optimism, which insist for 3 or 4 days, usually resulting in an increase in productivity and an improvement in performance more generally, other times we see a change in mood, which consists in the sudden transit from euthymia (or depression) to the state of mania, which can occur in a few hours, not infrequently after a sleepless night.

The state phase of mania has a duration on average lower than that of the depressive episode and is around 4-6 months. This is followed by the resolution phase, generally quite rapid (days, weeks), followed by a period of euthymia, a new depressive phase, or a mixed episode. In the state phase, the manic episode is defined as a defined period of abnormally and persistently elevated, expansive or irritable mood, lasting at least a week (or of any duration if hospitalization is necessary). During the period of mood alteration, three (or more) of the following symptoms were persistent and present at a significant level (four if the mood is only irritable) hypertrophic self-esteem or grandiosity; decreased need for sleep (e.g. feels rested after only 3 hours of sleep); greater talkativeness than usual, continuing to talk; escape of ideas or subjective experience that thoughts come quickly; distractibility (attention is easily diverted by external stimuli that are not important or irrelevant); increased targeted activity (social, work, school or sexual) or psychomotor agitation; involvement in recreational activities that have a high potential for damage (eg, excesses in buying, inappropriate sexual behavior, investments in rash business). The mood alteration is such as to compromise the working functioning or the usual social activities or interpersonal relationships or to require hospitalization to prevent damage to oneself or others, or psychotic manifestations are present and in any case the symptoms do not meet the criteria for the mixed episode and are not due to the direct physiological effects of a substance (e.g., a drug of abuse, a drug or other treatment) or a general medical condition (eg, hyperthyroidism). However, rather than from euphoria, the expanded mood is characterized by a specific widening of affective resonance and emotional lability, or by a substantial instability of mood. In the manic episode counter-oscillations are frequent: the patient, now playful, joyful, can quickly slip into a dark pessimism, to then return just as suddenly to the most immoderate hilarity. Moreover, although tendentially sociable and communicative, if contrasted it can easily become polemical, irascible, aggressive or even violent. Another characteristic of the manic episode is the acceleration of the ideational flow, which is the product of facilitation of associative processes. On the other hand, if at a moderate level of excitement the associations respect the elementary principles of logic, in a moment of later gravity we can find the so-called loosening of the associative links: the links between the ideas tend to become more superficial, perhaps taking place by rhyme or by phonetic similarity. In the most severe forms, ideational acceleration and loosening of the associative connections can come to configure the so-called flight of ideas (or fleeting thought) or the loss of directionality. In the extreme degrees of the escape of ideas, any residual link of concatenation is lost, which configures the so-called "unstitched thought" and which results in the peculiar "salad of words". In the most serious forms of mania, the relief of a reduction in attention capacity is frequent: the focus moves continuously and is often captured by secondary or irrelevant stimuli with respect to the set objectives. In fact, this marked distractibility is a reason for the profound disruption of all activities carried out. The rich ideational production of the manic patient converges on some contents with an expansive imprint: the vainglorious projects, the themes of greatness, the braggadocio, the ludic-fantastic fabulation are frequent. In some cases the themes of greatness take on delirious intensity: the delusions of ambition (the patient believes he possesses remarkable psychic or physical qualities); delusions of reform (the patient believes he can revolutionize the existing socio-political or religious structure); the inventor delusions (the patient is credited with inventions or ingenious discoveries); genealogical delusions (the patient is convinced of descending from an illustrious genealogy); power delusions (identifies with influential characters); the megalomaniac delusions (the patient is convinced that he possesses extraordinary psychophysical powers, sometimes even considering himself immortal); mystical-religious delusions (the patient would enjoy privileged contact with the divine); the delusions of enormity (the patient believes he has an immense, immortal and totipotent body, which often places at the centre of the universe); the delusions of love or Esquirol's erotomania or De Clérembault's imaginary lover's syndrome (the patient is convinced he is loved by a person who is actually ignorant); the delusions of infidelity (or jealousy), or the unfounded conviction of being betrayed by the partner (to this conclusion, that if in substance can also coincide with the truth, the patient arrives in a paralogical manner and in the absence of incontrovertible proof).

The craze also manifests itself on a volitional and motivational level, characterized by a marked increase in the initiative, or an exasperated push towards action. In fact, if the inhibition represents the behavioural outcome characteristic of depression, in mania, a specular counterpart, the diagnosis is often made possible by the manifestations of disinhibition and behavioural activation. The patient in a manic state shows an accentuated, hyper-expressive, theatrical mimicry and gestures, associated with a lively 
and accelerated motility, which can oscillate between simple motor restlessness and frank excitement. Speech, rapid and sustained, is often interrupted by exclamations, foul language, puns and daring jokes. The increased pressure of language ("push to speak"), which manifests itself with talkativeness, prolixity, logorrhea, can go so far as to configure the socalled "river of words". In such circumstances, the patient tends to slip of digression into a progressive course of drift. The ideo-verb-motor festination is part of a more general tendency to hyperactivity. This, if not exaggerated, can actually lead to an increase in productivity and performance but, when too strong, it implies progressive disorganization of behaviour.

Among the behavioral manifestations observable in the mania we also have the increase in planning, the involvement in high-risk activities, associated with the underestimation of the possible negative consequences, the lavishness, or the tendency to make unnecessary or excessive expenses, the abuse of sedatives and psychostimulants, verbal and / or physical aggressiveness, which can lead to clastic or pantoclastic crises of manic fury, such as heater direct violence, intrusiveness and other inappropriate behavior in interpersonal relationships: the patient tends to become inappropriate and deprived retention, showing uninhibited, vulgar and sometimes sexually promiscuous. On the somatic and neurovegetative level, the sense of physical well-being, the reduced sensitivity to heat and cold, the painful stimuli, the increase in energy levels, which is reflected in an apparent indefatigability, the reduced need for sleep, are characteristic. It can go as far as total insomnia, the accentuation of the libido, up to the "satyriasis" in the male and the "nymphomania" in the female.

From the manic episode, we must distinguish the "hypomanic" episode that is a defined period of persistently elevated, expansive or irritable mood, which lasts continuously for at least 4 days, and which is clearly different from the usual non-depressed mood. During the period of mood alteration three (or more) of the following symptoms were persistent and present at a significant level (4 if the mood is only irritable): hypertrophic or grandiose self-esteem; decreased need for sleep (e.g., feeling rested after only 3 hours of sleep); greater talkativeness than usual, or continuous push to speak; escape of ideas or subjective experience that thoughts come quickly; distractibility (i.e., attention is easily diverted by external stimuli that are not important or irrelevant); increased targeted activity (social, work, school or sexual), or psychomotor agitation; excessive involvement in recreational activities that have a high potential for harmful consequences (eg, excesses in buying, inappropriate sexual behavior, investments in rash business). Moreover, the hypomanic episode is associated with an evident change in the way of acting, which is not characteristic of the person when he is asymptomatic, and the alteration of mood and the change in the way of acting must be observable by others. However, however, the episode is not severe enough to cause marked impairment in the workplace or in society or to require hospitalization. However, the symptoms must not be due to the direct physiological action of a substance (e.g., abuse of a drug, drug or other treatment), or a general medical condition (e.g., hypothyroidism) and must not be present psychotic manifestations.

The hypomanic episode, however, must not be confused with the "free intervals", that is with the days of euthymia that follow the resolution of a major depressive episode. The discrimination between mania and hypomania is actually entirely artificial because it is based on poorly quantifiable criteria of severity and duration: so that one can diagnose a "hypomania", it is for example. It is necessary that the condition lasts for at least 4 days, which excludes all those hypomanic (documented) episodes lasting between 1 and 3 days, inevitably leading to an underestimation of the phenomenon. For mania, on the other hand, a duration of at least one week is required, as long as the severity of the condition is not such as to require hospitalization, in which case a shorter duration is sufficient. It is clear that this is a somewhat arbitrary conventional diagnostic threshold.

A further distinction must be made with respect to episodes of "melancholy", consisting of single depressive episodes of mild or moderate intensity, with or without delusional psychotic symptoms. It is by far the most frequent mood disorder, distinct from "major depression" which only represents the mode of onset of a more complex affective disorder. During the depressive episode, a debut phase can be identified, which is more often progressive. The onset is followed by the so-called state phase, which in the subject not subjected to any treatment shows an overall average duration of 6-8 months and is characterized by greater expressiveness of symptoms. The state period finally hesitates in the resolution phase, which can be more rapid than the onset and is often heralded by the normalization of the pattern. In its state phase, this condition is well described by DSM-V diagnostic criteria for the major depressive episode:

A) Five (or more) of the following symptoms were simultaneously present during a 2-week period and represent a change from the previous level of functioning; at least one of the symptoms consists of 1) depressed mood or 2) loss of interest or pleasure:

1. Depressed mood for most of the day, almost every day, as reported by the subject (e.g., feels sad or empty) or as described by others (e.g., appears plaintive). In children and adolescents the mood can be irritable;

2. A marked decrease in interest or pleasure (anhedonia) for all, or almost all activities, for most of the day, almost every day (as reported by the subject or as observed by others);

3. Significant weight loss, without being on a diet, or gaining weight (e.g., a change of more than $5 \%$ of body weight in a month), or decreased or increased appetite almost every day;

4. Insomnia or hypersomnia almost every day;

5. Agitation or psychomotor slowing almost every day (observable by others, not merely subjective feelings of being restless or slowed down);

6. Fatigue or lack of energy almost every day (asthenia and adynamia);

7. Excessive or inappropriate feelings of self-depreciation and guilt (which can be delusional), almost every day (not merely selfaccusation or feelings of guilt for being sick);

8. Reduced ability to think or concentrate, or indecision, almost every day (as a subjective impression or observed by others);

9. Recurrent death thoughts (not just fear of dying), recurrent suicidal ideation without a specific plan, or suicide attempt, or the creation of a specific plan to commit suicide.

B) The symptoms do not meet the criteria for a mixed episode.

C) Symptoms cause clinically significant distress or impairment in social, occupational, or other vital areas.

D) The symptoms are not due to the direct physiological effects of a substance (e.g., a drug of abuse, a medicine) or a general medical condition (e.g., hypothyroidism).

E) The symptoms are not better justified by mourning, that is, after the loss of a loved one, the symptoms persist for more than 2 months, or are characterized by a marked functional impairment, pathological selfdepreciation, suicidal ideation, psychotic symptoms or psychomotor slowing.

Very often, it is the patient himself who feels the difference between normal sadness and depression: in fact, the depressed mood is more intense, persistent, fixed and is scarcely sensitive to external stresses. In some cases the patient passes from a prevailing idea to a frank delirium that is to the firm conviction, sustained with stubborn tenacity despite every proof to the contrary, which configures the so-called psychotic depression. Generally, the content of delusions will tend to be congruous, that is, in line with the sufficient polarity, as in the mania there are megalomaniac delusions, in the depressive phase are characteristic for example delusions of guilt or selfaccusation (the patient can be held responsible for acts committed or due to venial shortcomings), the deliriums of poverty, poverty or ruin (the patient is convinced that he is economically compromised or has fallen into disgrace), hypochondriacal delusions (which consist in the absolute conviction of being affected by a terrible disease, in the absence of objectively detectable somatic lesions, up to trespassing on somatoform disorders, psychosomatic conversion disorder, hallucinatory parasitosis, etc.

The bipolar patient can reach, in the depressive phase, even beyond the "melancholic depression", up to the "catatonic depression", a rare and 
severe form of major depression that mainly involves disorders of the motor behavior and other symptoms (apathy, abulia, dysphoria, dysthymia), rather than real sadness. The individual appears dumb, sometimes even dysarthric (not aphasic) and almost soporous, remains motionless or shows movements without a purpose or even bizarre, with sometimes rigid musculature and mutacism (difficulty on the part of the delicate subject in pronouncing the labial consonants). Catatonic symptoms also occur in schizophrenia or during manic episodes or can be caused by neuroleptic malignant syndrome, and even only accentuated by some (iatrogenic) drugs, more efficiently during the depressive phase: other symptoms are plastic stiffness, automatic repetition of words (echolalia), in some cases the immobility is suddenly interrupted by strong tremors, agitations (akathisia) and sometimes escapes. Posture is fixed, stereotyped movements, echopraxia is present. The patient takes on the appearance of a statue, with immobility, inexpressiveness, inaccessibility. Due to the possibility of confusion, care must be taken in the diagnosis since in psychomotor depression mimicry is slowed down but remains fixed on a suffering expression, unlike parkinsonism which has an inexpressive mimic and superior stiffness; it is necessary to make a differential diagnosis with iatrogenic parkinsonism in bipolar patients in the depressive phase using neuroleptics, or with neuroleptics and antidepressants if they present only akathisia. However, this inactivity is only apparent, as it is instead supported by an intense commitment to negativist opposition, which can range from the effort of muscular tension to silence and the refusal to eat. Catalytic phenomena are also frequent in catatonic depression. In the case of acute and delusional manic episodes, it is more likely that the following depressive episode is severe.

\subsection{Differential diagnosis and evolutionary hypothesis borderline personality disorder [1]}

Several psychopathologies have symptoms of bipolar disorder in common; however, some differences characterize the other syndromes:

1. "bipolarity" differs from "manic disorder" because it also presents the depressive component; [23]

2. "bipolarity" differs from "major depressive disorder" because it also has the manic component [23];

3. "bipolarity" differs from "borderline personality disorder" mainly due to the degree of pervasiveness in the subject's psychic sphere. Nothing strange if the borderline was simply the psychopathological evolution from a mood disorder to personality disorder, even if the scientific community struggles to hypothesize this possibility, frankly plausible and less complex than the comorbidity hypothesis, considering them as two distinct entities and separate. [1-2] Beyond these speculations, however, there is no doubt the existence of a borderline clinic, characterized by severe difficulty in regulating impulsiveness and emotion, swings in mood, irritability and anger, symptoms psychotic (paranoid ideation, dissociative states) and self-injurious behaviours. Often pictures of this kind appear since early youth and remain rather stable over time, thus being rubricated by psychiatry as pervasive personality disorders. They are associated with a very high frequency to childhood traumas, and abuse, maltreatment, important emotional deficiencies suffered in childhood. The theme of abandonment is central and is the background of any typical dysfunctional behaviour. Bipolar disorder is, therefore, a mood disorder, so everything that happens to those who are subject can be completely dystonic with respect to his personality. If the borderline is so chronically affected by certain behavioural disturbances to make one think of penetration of discomfort into the deepest layers of character, the bipolar is instead invested by the dysregulation of mood as by an unexpected wave and alien to his way of being. The person is transformed as if under the influence of a drug, and others do not recognize it anymore. Thus, if the difficulty of regulating the impulses is a constant in the life of the borderline, bringing it systematically to act rather than to think, in the depressed maniac it appears in an episodic manner (especially in the manic phase with real passages at the act), distancing oneself from the usual character matrix of the person. So also, mood disorders are different in the two syndromes. In the borderline one, the oscillation is frequent, and the cycles are short, they last a few days or a few hours. The overhang is usually reactive to something that has to do with the perception of rejection by the other. Minimal signs of disinterest rather than alleged frustrations or losses are magnified and dramatized. In bipolar disorder, on the other hand, the oscillations are more discontinuous and lasting and can occur unexpectedly, regardless of the external situation, as if they were real lightning bolts from a clear sky. The same goes for anger and irritability. The borderline is chronically nervous, always reactive to events, often even insignificant ones. While the bipolar is so only when it is strongly melancholy or revved up, that is when it is at the mercy of the humoral storm that attacks it without reason. On psychotic symptoms, there is an additional clarification to make, also because the differential diagnosis of schizophrenia also comes into play. In borderline, we frequently observe paranoid ideation or pseudo-delusional conviction of being victims of a bad Other. However, it never reaches the level of a systematized delusion. Also the dissociative symptoms (detachment or amnesia), characterized by the absence of connection in thought, in memory and the sense of identity, never lead to complete detachment from reality: the patient remains able to understand that something strange is happening to him. The bipolar instead can experience real hallucinations and delusions both in the manic and depressive phases. In the first, themes of omnipotence and grandeur will prevail, while in the second delusion of ruin. What differentiates these psychotic symptoms from those of schizophrenia is the peculiarity of the contents in line with the concomitant alteration of mood, they are always being closely associated with the change of mood and being circumscribed over time. Furthermore, what qualifies schizophrenia is that we do not find either in the bipolar or in the borderline is a marked and pervasive emotional blunting (coerced affectivity). [24]

4. "bipolarity" differs from the "attention deficit and hyperactivity" because it presents the deficit symptomatology represents the constellation and not the only essence of the pathology;

5. "bipolarity" differs from "psychotic disorders" because it presents psychotic characterizations that are incidental and do not present all the elements for definitive diagnosis;

6. "bipolarity" is different from the other "mood disorders" because the humoral characterizations are accessory components although still present, to the point of thinking also of comorbidity.

\section{The neural correlates in bipolar disorder [25-26]}

The limbic system of our brain represents the mood thermostat and is responsible for keeping it stable and regular, ensuring that it responds adequately to stimuli and changes in the environment. In bipolar disorder, the limbic system does not work correctly, and this causes the mood to become variable, unstable and independent of the environment.

Bipolar disorder is often characterized by significant impairment of social and interpersonal functioning. Several recent neuroimaging studies have examined one of the main domains of social cognition, the understanding of emotions, in an attempt to identify the possible neurobiological substrate.

From the analysis of the results, an overall deficit of control of the cortical structures on the hyper-responsive limbic structures during the social interaction in the subjects with DB would emerge, which involves deficit of state-dependent emotional recognition (depression-mania). Such deficits would persist, however, even during disease-free phases (trait-dependence). Furthermore, the hippocampal neurons of people with bipolar disorder are over-excitable and respond with strong activation even to stimuli that do not cause reactions in healthy subjects' neurons.

\section{Clinical strategies for the management of the disorder} [27-34]

The best treatment suggested in the scientific literature is the one combined between psychopharmacology and psychotherapy.

The bipolar disorder is, in fact, with psychotropic drugs, such as mood tone stabilizers, which avoid the recurrence of mood swings. The best to date is lithium carbonate, in particular for the treatment of mania. However, anticonvulsant drugs are also used which have the effect of stabilizing moods, such as sodium valproate (useful in the treatment of mania and mixed states), carbamazepine (useful in the treatment of mania) and 
lamotrigine (useful in the prevention of mania). Depressive relapses); in particular, for the latter case, the molecule has a prevalent function of preventing depressive relapses, so much so that it is classified by some authors as an antidepressant. The mechanism by which all these drugs are able to stabilize the mood is not yet visible. In some cases, finally, anticonvulsants can lead to better results than lithium (and can be associated with it). Even atypical antipsychotics have an enormous effect on mood stabilization; among these are olanzapine, risperidone, quetiapine and clozapine.

In addition to drug therapy, psychotherapy can be useful and very helpful to those suffering from this disorder. It should be noted, however, that psychotherapy cannot replace drugs and is not sufficient alone in preventing relapses and treating the disorder. Many studies, however, have indicated that psychological interventions can improve mood stability, reduce hospitalization and improve functioning. Among the most suitable are suggested cognitive-behavioural and strategic treatment.

It should be noted, however, that no definitive cure is known for bipolar disorder; this is because the success of the therapy is directly related to the availability of the patient but also in the environment in which the patient lives. It is true that often the patient refuses treatment, as he does not recognize that he is sick or suspends them once discharged, but it is also true that those who have bipolar disorder are particularly vulnerable to the stress of the environment in which they live.

\section{Conclusions}

Bipolar disorder is classified as a mood disorder with necessary reflexes in the behavioural and personality sphere, such as to hypothesize that borderline personality disorder can be considered an evolution of bipolar disorder itself. However, the studies on the subject are very little focused on this hypothesis and targeted cross-sectional analyzes would be needed.

\section{References}

1. Perrotta G., Psicologia clinica, Luxco Ed., 1th ed., 2019.

2. Perrotta G., Psicologia generale, Luxco Ed., 1st ed., 2019.

3. Kessler M.C. (1994) Lifetime and 12-month prevalence of DSM III-R disorder in the United States: results from the national comorbidity Survey, in Arch gen Psychiatry, vol. 51, pp. 355-364.

4. Perlis R.H., Miyahara S., Marangell L.B., Wisniewski S.R., Ostacher M., DelBello M.P., et al. (2004) Long-term implications of early onset in bipolar disorder: data from the first 1000 participants in the Systematic Treatment Enhancement Program for Bipolar Disorder (STEP-BD). Biological Psychiatry. 55:875-881.

5. Monitor M. (2010) [Bipolar disorder in the elderly], in Vertex, vol. 21, no 92, pp. 275-83, PMID 21188315.

6. Goldberg T.E., Kotov R., Lee A.T., Gregersen P.K., Lencz T. (2009) The serotonin transporter gene and disease modification in psychosis: Evidence for systematic differences in allelic directionality at the 5-HTTLPR locus., in Schizophr Res., vol. 111, pp. 103-8.

7. Daban C., Vieta E., Mackin P., Young A.H., (2005) Hypothalamic-pituitary-adrenal axis and bipolar disorder, in Psychiatr Clin North Am., vol. 28, Giugno, pp. 469-480.

8. Crowe R.R., Vieland V., Report of the Chromosome 5 Workshop of the Sixth World Congress on Psychiatric Genetics, in Am J Med Genet., vol. 88, 1999, pp. 229-232.

9. Kato, T. (2007). "Molecular genetics of bipolar disorder and depression", Psychiatry Clin Neurosci 61(1): 3-19. PMID 17239033.

10. Reich, T., P. J. Clayton and G. Winokur (1969). "Family history studies-V The genetics of Mania", American Journal of Psychiatry, 125: 1358-1369.

11. Baum, A.E. et al. (2008). A genome-wide association study implicates diacylglycerol kinase eta (DGKH) and several other genes in the aetiology of bipolar disorder, Molecular Psychiatry, 13(2), 197-207.

12. Burton, P.R., et al. (2007). Genome-wide association study of 14,000 cases of seven common diseases and 3,000 shared controls, Nature, 447(7145), 661-678.

13. Sklar, P., J. W. Smoller, J. Fan, M. A. Ferreira, R. H. Perlis, K. Chambert et al. (2008), Whole-genome association study of bipolar disorder, Molecular Psychiatry

14. Ferreira, M., M. O'Donovan, Y. A. Meng, A. Jones I, D. M. Ruderfer1, L. Jones et al. (2008), Collaborative genome-wide association analysis supports a role for ANK3 and CACNA1C in bipolar disorder, Nature Genetics 40, 1056-1058.

15. Segurado R, Detera-Wadleigh S.D., Levinson D.F., Lewis C.M., Gill M., Nurnberger J.I. Jr, Craddock N., et al. (2003), Genome Scan Meta-Analysis of Schizophrenia and Bipolar Disorder, Part III: Bipolar Disorder. Am J Hum Genet. 73, 4962. PMID 12802785.

16. McQuillin, A., N. J. Bass, G. Kalsi, J. Lawrence, V. Puri, K. Choudhury, S. D. Detera-Wadleigh, D. Curtis and H. M. Gurling (2006). "Fine mapping of a susceptibility locus for bipolar and genetically related unipolar affective disorders, to a region containing the C21ORF29 and TRPM2 genes on chromosome 21q22.3." Mol Psychiatry 11(2): 134-142.

17. Xu, C., F. Macciardi, P. P. Li, I. S. Yoon, R. G. Cooke, B. Hughes, S. V. Parikh, R. S. McIntyre, J. L. Kennedy and J. J. Warsh (2006). "Association of the putative susceptibility gene, transient receptor potential protein melastatin type 2, with bipolar disorder." Am J Med Genet B Neuropsychiatr Genet 141(1): 36-43.

18. Barrett T.B., Hauger R.L., Kennedy J.L., Sadovnick A.D., Remick R.A., Keck P.E., McElroy S.L., Alexander M., Shaw S.H., Kelsoe J.R., Evidence that a single nucleotide polymorphism in the promoter of the $\mathrm{G}$ protein receptor kinase 3 gene is associated with bipolar disorder, in Molecular Psychiatry, vol. 8, $\mathrm{n}^{\circ}$ 5, may 2003, pp. 546-57, DOI:10.1038/sj.mp.4001268, PMID 12808434.

19. Zandi P.P., Belmonte P.L., Willour V.L., Association study of Wnt signaling pathway genes in bipolar disorder, in Arch. Gen. Psychiatry, vol. 65, n⿳0 7, luglio 2008, pp. 785-93,

20. Young E., New gene linked to bipolar disorder, on New Scientist, 2006.

21. Frans, E., Sandin, S., Reichenberg, A., Lichtenstein, P., Langstrom, N., Hultman, C. (2008), Advancing Paternal Age and Bipolar Disorder, Arch Gen Psychiatry. 2008;65(9):10341040.

22. Serretti A., Mandelli L. (2008), The genetics of bipolar disorder: genome 'hot regions,' genes, new potential candidates and future directions. Mol Psychiatry. 2008 August;13(8):74271. PMID 18332878.

23. Tondo L. Depression and mania in bipolar disorder. Curr Neuropharmacol. 2017 Apr; 15(3): 353-358.

24. Paris J., Black D.W., Borderline personality disorder and bipolar disorder: what is the difference and why does it matter? J Nerv Ment Dis., 2015 Jan; 203(1): 3-7.

25. Turchi F. et al., Neural basis of social cognition in bipolar disorder. Rivista di Psichiatria, Riv Psichiatr 2016; 51(5): 177189.

26. Mertens J., Wang Q.W., Differential responses to lithium in hyperexcitable neurons from patients with bipolar disorder. Nature 527, 95-99 (2015).

27. Solé B. Cognitive Impairment in Bipolar Disorder: Treatment and Prevention Strategies, Int J Neuropsychopharmacol. 2017 Aug 1;20(8):670-680.

28. Van der Markt A. et al., Exploring the clinical utility of two staging models for bipolar disorder. Bipolar Disord, 2019 Aug 26.

29. Vieta E, Franco C., Advances in the treatment of mania: aripiprazole, in Actas Esp Psiquiatr., vol. 36, may 2008, pp. 158-164. 
30. Mallinger G., Frank E., Thase M. E., Revisiting the Effectiveness of Standard Antidepressants in Bipolar Disorder: Are Monoamine Oxidase Inhibitors Superior?, in Psychopharmacology bulletin, vol. 42, no 2, 2009, pp. 6474.

31. Bersani F.S., Girardi N., Sanna L. et al, Deep Transcranial Magnetic Stimulation for treatment-resistant bipolar depression: A case report of acute and maintenance efficacy., in Neurocase, PMID 22827578.
32. Kennedy D., Koren G., Valproic acid use in psychiatry: issues in treating women of reproductive age. J Psychiatry Neurosci. 1998 Sep;23(4):223-8. PMID 9785701.

33. Behzadi A. H., et al., Folic acid efficacy as an alternative drug added to sodium valproate in the treatment of acute phase of mania in bipolar disorder: a double-blind randomized controlled trial. Acta Psychiatr Scand 2009: 1-5.

34. Colom C., Keeping therapies simple: psychoeducation in the prevention of relapse in affective disorders. Brithish Journal of Psychiatry, May 2011, 198:338-340. 